\title{
miR-101 regulates the cell proliferation and apoptosis in diffuse large B-cell lymphoma by targeting MEK1 via regulation of the ERK/MAPK signaling pathway
}

\author{
YIQUN HUANG，YONG ZOU，LUHUI LIN，XUDONG MA and RUIJI ZHENG \\ Department of Hematology, Zhangzhou Affiliated Hospital of Fujian Medical University, \\ Zhangzhou, Fujian 363000, P.R. China
}

Received February 27, 2018; Accepted September 27, 2018

DOI: 10.3892/or.2018.6821

\begin{abstract}
MAPK kinase 1 (MEK1) is an upstream protein kinase of extracellular signal regulated kinase (ERK), which activates the ERK/MAPK (mitogen activated protein kinase) pathway. Importantly, bioinformatic analysis has shown that there is a target complementary binding site between miR-101 and MEK1. The present study aimed to ascertain whether or not miR-101 plays a role in regulating MEK1 expression, ERK/MAPK pathway activity, and the proliferation and apoptosis in a diffuse large B cell lymphoma (DLBCL) cell line. DLBCL tumor samples were collected from patients in our hospital, and lymphatic tissues with reactive lymphoid hyperplasia were selected as controls. The patients were divided into high and low expression groups, and then the survival rate of the two groups was compared using Kaplan-Meier method, as well the effect of miR-101 and MEK1 mRNA expression on survival and prognosis was analyzed. The expression of miR-101, MEK1 and p-MEK1 between normal lymphoblastic cell lines (HCC1954 BL and NCI-BL2009) and lymphoma cell lines (SU-DHL-4 and Farage) was compared. Lymphoma SU-DHL-4 and Farage cells were cultured in vitro, and then divided into the following groups: miR-NC group; miR-101 mimic group; siRNA-NC group; and siRNA-MEK1 group. The expression of miR-101, MEK1, p-MEK1, p-ERK1/2 and Bcl-2 was compared. Cell apoptosis was detected by flow cytometry, and cell proliferation was detected by EdU staining. The results showed that targeted regulation existed between miR-101 and MEK1, and the decreased expression of miR-101 was related to the pathogenesis and prognosis of DLBCL. Upregulation of miR-101 inhibited DLBCL cell proliferation and facilitated apoptosis by inhibiting the expression of MEK1.
\end{abstract}

Correspondence to: Dr Ruiji Zheng, Department of Hematology, Zhangzhou Affiliated Hospital of Fujian Medical University, 59 Shengli Road, Zhangzhou, Fujian 363000, P.R. China

E-mail: ruijizheng0113@126.com

Key words: miR-101, MEK1, ERK/MAPK, diffuse large B cell lymphoma, proliferation, apoptosis

\section{Introduction}

Lymphoma is a malignant tumor that occurs in the lymphoid and hematologic systems, such as lymph nodes, spleen, thymus and extranodal lymphatic tissues and organs. Lymphomas are associated with immune cell malignant transformation, and thus are categorized as immune system malignant tumors $(1,2)$. Among the immune system malignant tumors, non-Hodgkin lymphoma (NHL) is the most commonly diagnosed type of lymphoma (3). Diffuse large B cell lymphoma (DLBCL) is the most prevalent type of $\mathrm{B}$ cell-derived non-Hodgkin lymphoma (B-NHL). DLBCL is also the most common NHL in adults $(4,5)$. DLBCL is an invasive lymphoma with a low rate of cure and poor prognosis.

Increased activation of the extracellular signal regulated kinase $(\mathrm{ERK}) / \mathrm{mitogen}$ activated protein kinase (MAPK) signal transduction pathway is closely related to the occurrence, development and metastasis of multiple cancers. MAPK kinase 1 (MEK1) is a double-specific protein kinase which can act upstream of ERK protein and phosphorylate the tyrosine/threonine (Tyr/Thr) residues of ERK protein, thereby activating the ERK/MAPK signaling pathway. A number of studies have shown that an abnormal increase in MEK1 expression or functional activity is related to the occurrence, progression, drug resistance and poor prognosis of numerous types of tumors, such as pancreatic, ovarian and lung cancers (6-8). Moreover, it has been shown that the expression or function enhancement of MEK1 plays an important role in lymphomas (9). MicroRNAs (miRNAs) are a type of small molecule non-coding RNAs with a length of 22-25 nucleotides that were initially discovered in eukaryotes. miRNAs can be adjusted to match the target gene mRNA 3 '-end untranslated region (3'-UTR) with complete or incomplete complementation, and can regulate the expression of $>30 \%$ of human target genes by degradation or inhibition of target gene mRNA translation. Depending on the targeting-specific genes, miRNAs are involved in the pathogenesis of tumors acting as oncogenes or tumor-suppressor genes (10-13). Previous studies have shown that the expression of miR-101 in colorectal (14), esophageal (15) and ovarian cancers (16) is abnormally reduced, suggesting that miR-101 may play a role as a tumor-suppressor gene in tumorigenesis. It has also been 
reported that the abnormal decrease in miR-101 expression is associated with the incidence of lymphomas, suggesting a possible tumor-suppressor effect $(17,18)$. Bioinformatic analysis showed a complementary binding site between miR-101 and the 3'-UTR of MEK1 mRNA. Therefore, in the present study we determined the role of miR-101 in regulating the expression of MEK1, the activity of the ERK/MAPK pathway, and lymphoma cell proliferation and apoptosis.

\section{Materials and methods}

Main reagents and materials. Normal human B lymphoblastoid cell lines (HCC1954 BL and NCI-BL2009) were purchased from the American Type Culture Collection (ATCC; Manassas, VA, USA). 293T cells were purchased from Hunan Fenghui Biotechnology Co., Ltd. (Hunan, China). Human DLBCL cell lines (SU-DHL-4 and Farage) were purchased from ATCC. Gibco ${ }^{\mathrm{TM}}$ RPMI-1640 medium, fetal bovine serum (FBS), penicillin and streptomycin were purchased from Thermo Fisher Scientific, Inc. (Waltham, MA, USA). The RNA extraction reagent, TRIzol, and the transfection reagent, Lipofectamine 2000, were purchased from Thermo Fisher Scientific, Inc. (Invitrogen ${ }^{\mathrm{TM}}$; Waltham, MA, USA). The PrimeScript RT reagent kit and SYBR Fast qPCR Mix were purchased from Takara (Dalian, China). Rabbit anti-human MEK1 (cat. no. 12671), p-MEK1 (cat. no. 98195) and Bcl-2 (cat. no. 4223) antibodies were purchased from Cell Signaling Technology, Inc. (Beverly, MA, USA). Mouse anti-human ERK1/2 (cat. no. ab36991), p-ERK1/2 (cat. no. ab126423) and PCNA antibodies (cat. no. ab70472) were purchased from Abcam (Cambridge, MA, USA). The Annexin V/PI apoptosis detection kit and Cell Cycle detection kit were purchased from Biyuntian (Jiangsu, China). siRNA-MEK1 and siRNA-NC were purchased from Santa Cruz Biotechnology (Santa Cruz, CA, USA). A cell proliferation detection kit (Click-iT ${ }^{\circledR}$ EdU Alexa Fluor 488 Flow Cytometry Assay kit) was obtained from Molecular Probes (Eugene, OR, USA). The Dual-Glo ${ }^{\circledR}$ Luciferase Assay System and pMIR luciferase reporter gene plasmid were provided by Promega (Madison, WI, USA).

Clinical data. A total of 72 patients diagnosed with DLBCL who received treatment at our hospital between September 2011 and April 2015 and had complete follow-up data were enrolled, including 38 males and 34 females (age range, 19-77 years; median age, 58 years; and average age, 50.7 years). Patients who did not undergo previous radiotherapy and chemotherapy were included, and patients who underwent antitumor treatment pre-operatively were excluded. The tumor tissue specimens were collected intra-operatively, immediately frozen in liquid nitrogen, and stored at $-80^{\circ} \mathrm{C}$. The tissue specimens were staged according to the Ann Arbor staging scheme as follows: I, invasion of a lymph node or an extranodal organ or part; II, diaphragmatic side, involving two or more lymph nodes or external limitations infringing on an extranodal organ or part; III, violation of both surfaces of the diaphragm, the junction, or additional limitations to infringe upon an extranodal organ or part or the spleen; and IV, diffuse invasion or disseminated extranodal organ or part of one or more. Among the participants, 42 were stages I-II and 30 were stages III-IV. The distribution of patients according to the Lymphoma International Prognostic Index (IPI) was as follows: $0-1, n=25 ; 2, n=22 ; 3, n=16$; and $4-5, n=9$. There were 28 patients with $\mathrm{B}$ symptoms, and 44 patients had no B symptoms.

The lymphatic tissue samples from 30 patients with reactive hyperplasia of lymph nodes were designated as the control group. All of the patients signed informed consent, which was approved by the Ethics Committee of Zhangzhou Affiliated Hospital of Fujian Medical University (protocol no. 2010032).

Cell culture. HCC1954 BL, NCI-BL2009, SU-DHL-4, and Farage cells were cultured in RPMI-1640 medium containing $10 \%$ FBS and $1 \%$ penicillin-streptomycin. The cells were passaged at a 1:4 ratio, and cells with suitable growth in the logarithmic phase were used in all experiments.

Construction of the recombinant plasmid with double luciferase gene report. The whole genome DNA of 293T cells was used as a template, and PCR amplification contained the MEK1 3'-UTR region of the miR-101 binding site. SacI and HindIII enzyme cutting sites and protective bases were added to the 5'-terminal of the upstream and downstream primers, respectively. The primer sequences for MEK1 were as follows: Sense, 5'-CGAGCTCAAGCAACAAAGAGC GAGTCCCCT-3' and antisense, 5'-CCAAGCTTGCAAAGC ATGCTTCACATGCACT-3' (SacI and HindIII enzyme sites are underlined; the former is a protective base). The amplified product was the 10-465 bp range of the 3'-UTR region of MEK1. The total PCR amplification reaction volume consisted of the following: 2X KOD buffer $10 \mu \mathrm{l}$; d NTP $(2 \mathrm{mM}) 4 \mu \mathrm{l}$; primer $\mathrm{F}(10 \mu \mathrm{M}) 0.5 \mu \mathrm{l}$; primer $\mathrm{R}(10 \mu \mathrm{M}) 0.5 \mu \mathrm{l}$; KOD FX $0.4 \mu \mathrm{l}$; template DNA $2 \mu \mathrm{l}$; and $\mathrm{ddH}_{2} \mathrm{O} 3.6 \mu \mathrm{l}$. The cycling conditions were as follows: $95^{\circ} \mathrm{C}$ for $10 \mathrm{~min}$, followed by 30 cycles at $94^{\circ} \mathrm{C}$ for $30 \mathrm{sec} ; 58^{\circ} \mathrm{C}$ for $30 \mathrm{sec}$; and $72^{\circ} \mathrm{C}$ for $10 \mathrm{~min}$, with $4^{\circ} \mathrm{C}$ infinity. After PCR amplification, SacI and HindIII double enzymes were used to cut the PCR products and pMIR Noblets. The conditions for the enzyme digestion were $37^{\circ} \mathrm{C}$ for $4 \mathrm{~h}$. The purified product was recovered by $1.5 \%$ agarose gel electrophoresis, and the purified PCR products were incubated at $16^{\circ} \mathrm{C}$ for overnight connection. The products were transformed into DH5 $\alpha$ competent cells, inoculated on plates containing penicillin, and incubated at $37^{\circ} \mathrm{C}$ overnight. Then, a single positive clone was selected and incubated in Escherichia coli culture medium at $37^{\circ} \mathrm{C}$ overnight to extract the plasmids. The sequence was identified, and designated as pMIR-MEK1-WT. Using the wild-type plasmid as a template, miR-101 and MEK1 3'-UTR binding region mutations for meaningless sequence design of MEK1 point mutation primers were as follows: forward, 5'-CTCTTG TGTTAATAAATATTACTGTCT-3' and reverse, 5'-AAGTGT CATATATTAAAAATGACAAGA-3' (mutant sequences are underlined). The detailed construction steps were the same as the wild-type, and the constructed mutant plasmid was designated as pMIR-MEK1-MUT.

Dual-luciferase reporter gene assay. The $293 \mathrm{~T}\left(1 \times 10^{5}\right)$ cells were inoculated in 24-well plates. After being cultured in DMEM containing 10\% FBS for $24 \mathrm{~h}$, the cells were divided into 4 transfection groups: pMIR-MEK1-WT+miR-101 mimic group; pMIR-MEK1-WT+miR-NC group; 
pMIR-MEK1-MUT+miR-101 mimic group; and pMIR-MEK1MUT+miR-NC group. Each group had 3 duplicate pores. The specific transfection additions were as follows: $0.8 \mu \mathrm{g}$ of gene recombinant plasmid; $0.02 \mu \mathrm{g}$ of pRL-SV40 plasmid; and 20 pmol miR-101 mimic or miR-NC in $50 \mu 1$ of Opti-MEM. The mixture was then stored for $5 \mathrm{~min}$ at room temperature. Lipofectamine $^{\mathrm{TM}} 2000(2 \mu \mathrm{l})$ was added to $50 \mu \mathrm{l}$ of Opti-MEM, and was placed at room temperature for $5 \mathrm{~min}$ after gentle mixing. The two mixtures were combined after mixing for $20 \mathrm{~min}$ at room temperature. The 4 groups of cells were added to 24-hole plates. DMEM fresh medium was replaced by culture medium at $37^{\circ} \mathrm{C}$ for $6 \mathrm{~h}$, and the cultures were continued for $48 \mathrm{~h}$. A Dual-Glo Luciferase Assay System kit was used to detect luciferase activity, as follows: $100 \mu \mathrm{l}$ of Passive Lysis Buffer cell lysate was added to each hole of a 24-well plate; the plates were slowly shaken at room temperature for $15 \mathrm{~min}$; $20 \mu 1$ of cell lysate was added to $100 \mu 1$ of luciferase assay reagent II (LAR II), mixed, and the fluorescence of the firefly luciferase activity was detected. Fluorescein $(100 \mu l)$ was added to the detection kit. The Renilla luciferase activity of firefly luciferase activity to Renilla luciferase activity/relative activity value was analyzed.

Cell transfection and grouping. The day before transfection, SU-DHL-4 and Farage cells were cultured in 10-cm culture dishes, ensuring that the transfection cell density was 50-60\% on the day of fusion, and the transfected cells were divided into the following 4 groups: miR-NC group; miR-101 mimic group; siRNA-NC group; and siRNA-MEK1 group. The miR-NC, miR-101 mimic, siRNA-NC and siRNA-MEK1 group cells were diluted with Opti-MEM and incubated at room temperature for $5 \mathrm{~min}$. Each transfected nucleotide was then mixed with Lipofectamine 2000 and gently inverted by mixing, and incubated for $30 \mathrm{~min}$ at room temperature. The cell culture medium was removed and washed twice in PBS to remove serum, and then replaced with Opti-MEM serum-free cell culture medium. The transfection complexes were added to the culture medium and mixed. After culturing for $6 \mathrm{~h}$, they were replaced with RPMI-1640 medium containing 10\% FBS and $1 \%$ penicillin. After being cultured for $72 \mathrm{~h}$, they were trypsinized. Cells were harvested for gene, protein expression, and flow cytometric assays.

qRT-PCR detection of gene expression. One milliliter of TRIzol was added to every $20 \mathrm{mg}$ of tissue or every $3 \times 10^{6}$ cells. After full lysis, the tissues or cells were added to chloroform for extraction. After stratification, the RNA supernatant was transferred to a new EP tube. After washing with isopropanol and 70\% ethanol, DEPC water was dissolved to obtain RNA. The RNA was reversed transcribed into cDNA using PrimeScript RT reagent kit with cDNA as the template and qPCR detecting gene expression. The reverse transcription reaction system contained $0.5 \mu \mathrm{l}(50 \mu \mathrm{M}), 0.5 \mu \mathrm{l}$ of random 6 mers $(100 \mu \mathrm{M}), 0.5 \mu \mathrm{l}$ of PrimeScript RT Enzyme Mix, $1.0 \mu \mathrm{g}$ of RNA, $2 \mu \mathrm{l}$ of $5 \mathrm{X}$ PrimeScript Buffer, and RNase-free $\mathrm{H}_{2} \mathrm{O}_{2}$ was added to a total volume of $10 \mu \mathrm{l}$. The reverse transcription conditions were $37^{\circ} \mathrm{C}$ for $15 \mathrm{~min}$ and $85^{\circ} \mathrm{C}$ for $5 \mathrm{sec}$. The PCR reaction system contained SYBR Fast qPCR Mix (10.0 $\mu \mathrm{l})$, $0.8 \mu 1$ of forward primer $(10 \mu \mathrm{M}), 0.8 \mu 1$ of reverse primer $(10 \mu \mathrm{M}), 2.0 \mu \mathrm{l}$ of cDNA, and $6.4 \mu \mathrm{l}$ of RNase-free $\mathrm{dH}_{2} \mathrm{O}$. The
PCR reaction consisted of $95^{\circ} \mathrm{C}$ pre-denaturation for $10 \mathrm{~min}$, followed by 40 cycles at $95^{\circ} \mathrm{C}$ for $5 \mathrm{sec}, 60^{\circ} \mathrm{C}$ for $20 \mathrm{sec}$, and extension at $72^{\circ} \mathrm{C}$ for $15 \mathrm{sec}$. Real-time PCR was performed on Bio-Rad CFX96/CFX Connect ${ }^{\mathrm{TM}}$ (Bio-Rad Laboratories, Inc., Hercules, CA, USA) to test the relative expression.

Western blot analysis. Total protein was extracted by RIPA from cells or tissues. A total of $40 \mu \mathrm{g}$ of protein was separated by $8-10 \%$ sodium lauryl sulfate-polyacrylamide gel electrophoresis (SDS-PAGE) and 5\% concentration gel for $3 \mathrm{~h}$ and transferred to nitrocellulose membranes. Next, the membranes were blocked with $5 \%$ skim milk at room temperature for $60 \mathrm{~min}$ and incubated with the primary antibody at $4^{\circ} \mathrm{C}$ overnight (MEK1, MMP-2, anti ERK1/2, ERK1/2, Bcl-2 and $\beta$-actin; the dilution ratios were $1: 2,000,1: 1,000$, 1:2,000, 1:1,000, 1:2,000, 1:10,000 and PBST, respectively). After thrice-washing, the membranes were incubated with horseradish peroxidase (HRP)-labeled secondary antibody $(1: 20,000)$ for $60 \mathrm{~min}$, then thrice-washed. Finally, protein expression was detected by enhanced chemiluminescence (ECL). Data were analyzed with ImageJ software k1.45 (National Institutes of Health, Bethesda, MD, USA).

Flow cytometry to detect cell proliferation. Each transfection group of cells was resuspended in complete medium. After a 120-min incubation with $10 \mu \mathrm{M}$ EdU, the cells were continuously cultured for $48 \mathrm{~h}$, and then the cells were collected after trypsinization. The cells were washed during $250 \mathrm{x}$ g centrifugation 1 time with PBS containing 1\% BSA, and then $100 \mu \mathrm{l}$ of Click-iT fixative was added for $15 \mathrm{~min}$ and washed during $250 \mathrm{x}$ g centrifugation 1 time with PBS containing 1\% BSA. The cells were added to $100 \mu \mathrm{l}$ of $1 \mathrm{X}$ Click-iT saponin-based permeabilization for $15 \mathrm{~min}$ at room temperature and $500 \mu \mathrm{l}$ of PBS, $\mathrm{CuSO}_{4}$, Alexa Fluor 488, and buffer was added before incubation in the dark at room temperature for $30 \mathrm{~min}$. The cells were added to $3 \mathrm{ml}$ of $1 \mathrm{X}$ Click-iT saponin-based permeabilization and washed during $250 \mathrm{x}$ g centrifugation 1 time. The cells were then added to $500 \mu \mathrm{l}$ of $1 \mathrm{X}$ Click-iT saponin-based permeabilization and wash reagent and tested on a Beckman Kurt FC 500 MCL/MPL flow cytometry device (Beckman Coulter, Inc., Brea, CA, USA) to evaluate cell proliferation.

Flow cytometry to detect cell apoptosis. The transfected cells were digested by trypsin and collected after $250 \mathrm{x}$ g centrifugation, then washed 2 times in PBS. Cells were incubated in $5 \mu \mathrm{l}$ of Annexin V-FITC and $5 \mu \mathrm{l}$ of PI in the dark for $10 \mathrm{~min}$. Next, the cells were resuspended in $400 \mu 1$ of binding buffer and tested on a Beckmann FC 500 MCL/MPL flow cytometry device to evaluate cell apoptosis.

Statistical analysis. All data analyses were performed using SPSS 18.0 software (SPSS, Inc., Chicago, IL, USA). The measurement data are depicted as the mean \pm standard deviation and were compared by t-tests. For two-group comparisons, a Chi-square test was used, and when making comparisons among multiple groups, one way ANOVA test was used to determine the relationship between the level of miR-101 expression and the clinical characteristics of the patients. The Mann-Whitney U test was used to compare the level 
of miR-101 and MEK1 mRNA expression in the lymphoid tissues of the two groups. The survival curve was drawn with the Kaplan-Meier method, and the survival rate was compared using a log-rank test. A $\mathrm{P}<0.05$ was considered statistically significant.

\section{Results}

miR-101 targeted regulation of MEKI expression. MicroRNA. org online prediction showed the targeted binding site between miR-101 and 3'-UTR of MEK1 mRNA (Fig. 1A). A dual luciferase assay revealed that miR-101 mimic transfection significantly decreased the relative luciferase activity of 293T cells transfected with wild-type pMIR-MEK1-WT, while the relative luciferase activity of 293T cells transfected with mutant pMIR-MEK1-MUT was not affected (Fig. 1B), indicating the regulatory relationship between miR-101 and MEK1.

miR-101 is reduced, while MEK1 is increased in DLBCL tissues. Based on quantitative RT-PCR (qRT-PCR), it was shown that the expression of miR-101 in tumor tissues from DLBCL patients was significantly decreased (Mann-Whitney $\mathrm{U}$ value $=367, \mathrm{P}<0.05$ ) when compared with proliferative lymph node tissues in the control group (Fig. 2A), while the expression of MEK1 mRNA was elevated in the tumor tissues from DLBCL patients compared with the control group (Mann-Whitney $\mathrm{U}=269, \mathrm{P}<0.05$; Fig. 2A). Western blot analysis showed that the expression levels of MEK1 and p-MEK1 protein were significantly increased in the DLBCL tissues when compared with levels in the control tissues; representative illustrations are shown in Fig. 2B. Based on the median miR-101 expression, DLBCL patients were divided into a miR-101 high-expression group and a miR-101 low-expression group. The relationship between clinical features and the level of miR-101 expression was analyzed. According to $\chi^{2}$ test analysis, the number of patients with high expression of miR-101 was higher in patients with clinical stage I-II and the number of patients with low expression of miR-101 was significantly higher in patients with clinical stage III-IV $(\mathrm{P}=0.017)$. The proportion of patients with an IPI score $>3$ points was higher in the low miR-101 expression group and the proportion of patients with an IPI score $<3$ was significantly higher in the high miR-101 expression group $(\mathrm{P}=0.026)$, yet patient age, gender, no $\mathrm{B}$ symptoms, and miR-101 expression had no apparent relationship ( $\mathrm{P}>0.05$; Table I). Survival analysis showed that the survival and prognosis of patients with high expression of miR-101 were significantly better than the patients with low miR-101 expression (log-rank test $\chi^{2}=5.684, \mathrm{P}=0.017$ ). Survival and prognosis of patients with low expression MEK1 mRNA were better than patients with high expression of MEK1 mRNA (log-rank test $\chi^{2}=5.360, P=0.021$; Fig. 2C).

Overexpression of miR-101 downregulates ERK/MAPK pathway activity. qRT-PCR detection showed that compared with normal lymphoblastoid cells (HCC1954 BL and NCI-BL2009), the expression of miR-101 in SU-DHL-4 and Farage cells was significantly decreased and MEK1 mRNA expression was significantly increased (Fig. 3A). Western blot detection showed that compared with normal lymphoblastoid cells (HCC1954 BL and NCI-BL2009), the
A

3' aagUCAAUAGUGUCAUGACAu 5' hsa-miR-101 || || | |:| ||||||

$150: 5^{\prime}$ guCAUUUUUAAUAUUACUGUC 3' MAP2K1

B miR-NC $\square$ miR-101 mimic

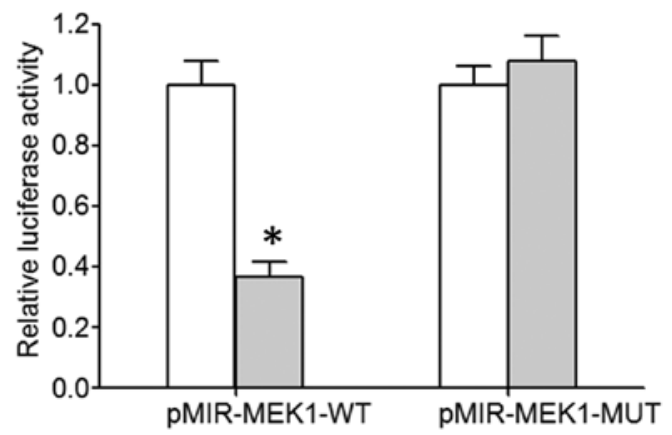

Figure 1. miR-155 targets the regulation of MEK1expression. (A) The binding site between miR-101 and the 3'-UTR of MEK1 mRNA. (B) Dual-luciferase assay. ${ }^{*} \mathrm{P}<0.05$, compared with miR-NC. UTR, untranslated region.

Table I. Relationship between the expression of miR-101 and clinical characteristics of the DLBCL cases.

\begin{tabular}{lccccc}
\hline & \multicolumn{5}{c}{$\begin{array}{c}\text { miR-101 } \\
\text { expression } \\
\text { level }\end{array}$} \\
\cline { 3 - 4 } Clinical features & $\mathrm{n}$ & High & Low & $\chi^{2}$ & P-value \\
\hline Age (years) & & & & 1.399 & 0.237 \\
$\leq 60$ & 33 & 19 & 14 & & \\
$>60$ & 39 & 17 & 22 & & \\
Sex & & & & 0.892 & 0.345 \\
Male & 38 & 21 & 17 & & \\
Female & 34 & 15 & 19 & & \\
Clinical stage & & & & 5.714 & 0.017 \\
I-II & 42 & 26 & 16 & & \\
III-IV & 30 & 10 & 20 & & \\
IPI score & & & & 4.963 & 0.026 \\
$\quad$ 0-2 & 47 & 28 & 19 & & \\
$3-5$ & 25 & 8 & 17 & & \\
$\quad$ B symptoms & & & & 2.104 & 0.147 \\
$\quad$ Present & 28 & 17 & 11 & & \\
Absent & 44 & 19 & 25 & & \\
\hline
\end{tabular}

DLBCL, diffuse large B cell lymphoma; IPI, International Prognostic Index.

expression of MEK1 and p-MEK1 protein in SU-DHL-4 and Farage cells was significantly increased (Fig. 3B). The expression of miR-101 in SU-DHL-4 and Farage cells was significantly increased in the miR-101 mimic transfection 
A
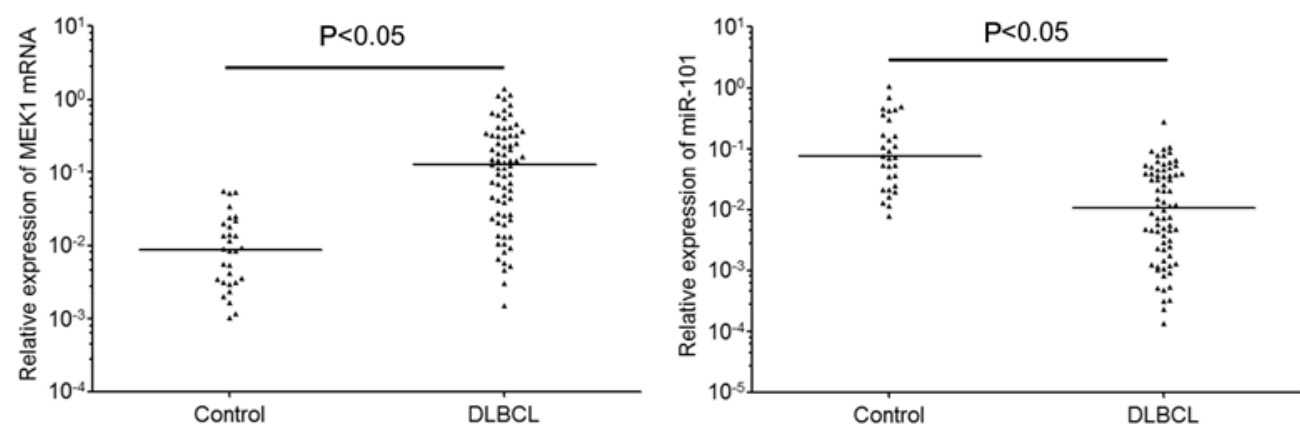

B

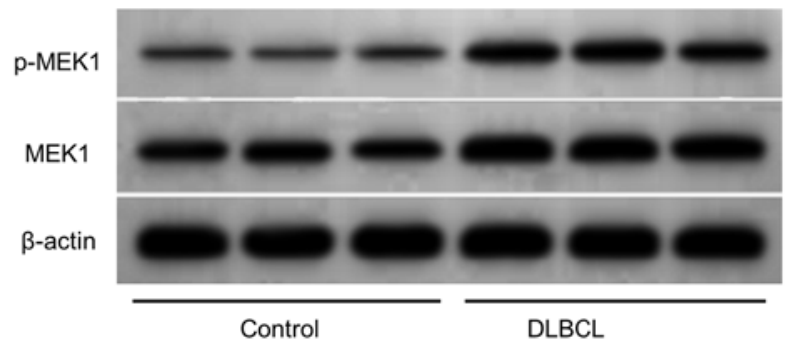

C
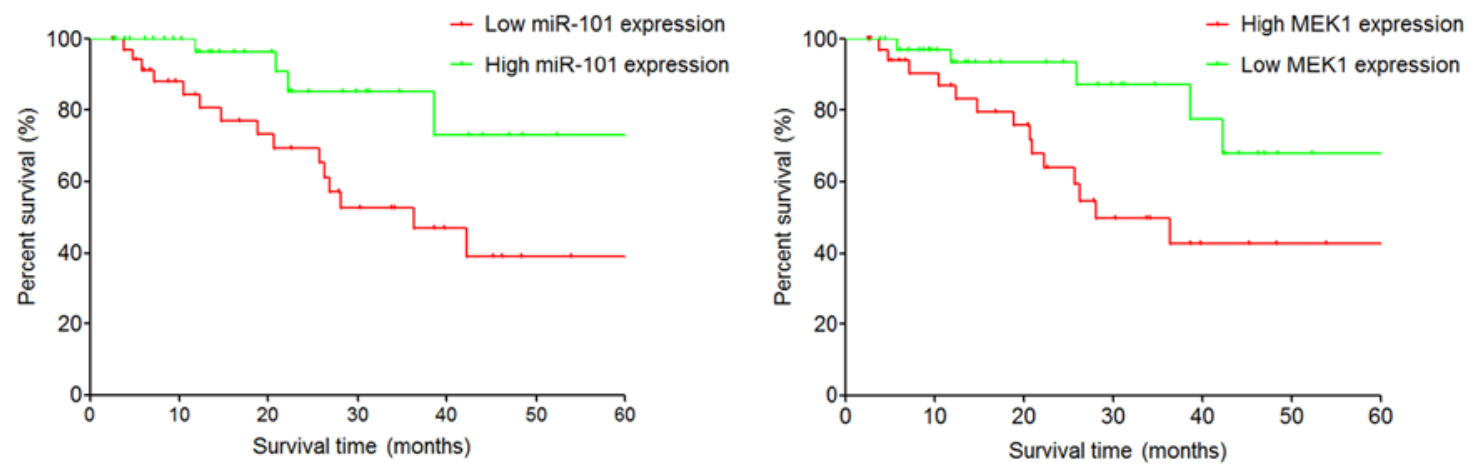

Figure 2. miR-101 is reduced, while MEK1 is increased in DLBCL tissues. (A) qRT-PCR detection of miR-101 and MEK1 mRNA expression in lymphoid tissues. (B) Western blot analysis of MEK1 and p-MEK1 protein expression in lymphoid tissues. (C) Survival curves of patients with high and low expression of miR-101 and MEK1 mRNA. DLBCL, diffuse large B cell lymphoma.

group compared with the miR-NC group while MEK1 mRNA expression was significantly decreased (Fig. 3C). Compared with the siRNA-NC group, the expression of MEK1 mRNA in the SU-DHL-4 and Farage cells of the siRNA-MEK1 transfected group was significantly decreased (Fig. 3C). Western blot analysis showed that transfection of miR-101 mimic or siRNA-MEK1 significantly reduced MEK1, p-MEK1 and p-ERK1/2 protein expression in the SU-DHL-4 and Farage cells (Fig. 3D).

Overexpression of miR-101 inhibits the proliferation of lymphoma cells. Flow cytometric analysis showed that the proliferative rate of SU-DHL-4 and Farage cells was significantly higher than the HCC1954 BL and NCI-BL2009 cells (Fig. 4A). The EdU staining flow test showed that the EdU-positive rate of SU-DHL-4 and Farage cells in the miR-101 mimic transfected group was significantly lower than the miR-NC group. Compared with the siRNA-NC group, the EdU-positive rates of SU-DHL-4 and Farage cells in the siRNA-MEK1 transfected group were significantly lower (Fig. 4B and C).

Overexpression of miR-101 promotes the apoptosis of lymphoma cells. Flow cytometric analysis showed that the apoptosis rate of SU-DHL-4 and Farage cells was significantly lower than that noted in the HCC1954 BL and NCI-BL2009 cells (Fig. 5A). The flow cytometry test showed that the apoptosis rate of SU-DHL-4 and Farage cells in the miR-101 mimic transfected group was significantly higher than that noted in the miR-NC group. Compared with the siRNA-NC group, the apoptosis rate of the SU-DHL-4 and Farage cells in the miR-101 minic and siRNA-MEK1 group was significantly higher (Fig. 5B and C). Using western blot analysis we found that miR-101 targets Bcl-2, PARP and caspase-3 expression to regulate the apoptosis of lymphoma cells. When miR-101 expression was increased, expression of the anti-apoptotic factor Bcl-2 protein was significantly decreased, and PARP and caspase-3 expression was increased (Fig. 5D). 


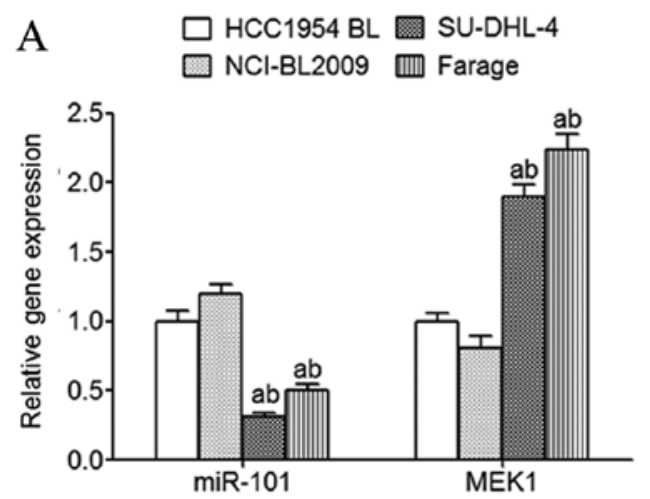

B

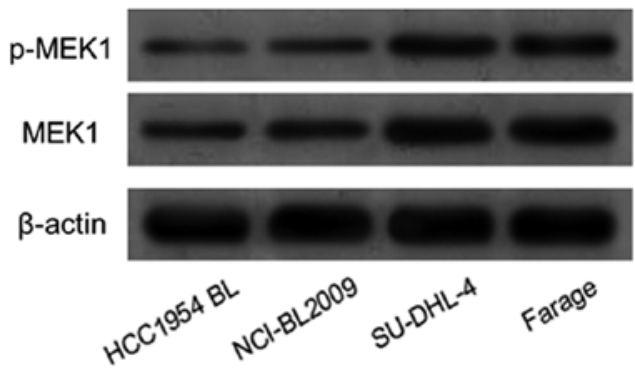

$\mathrm{C}$
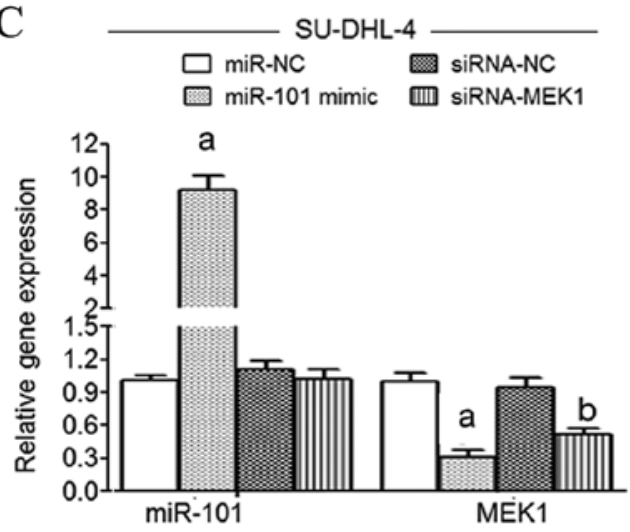

$\mathrm{D}$
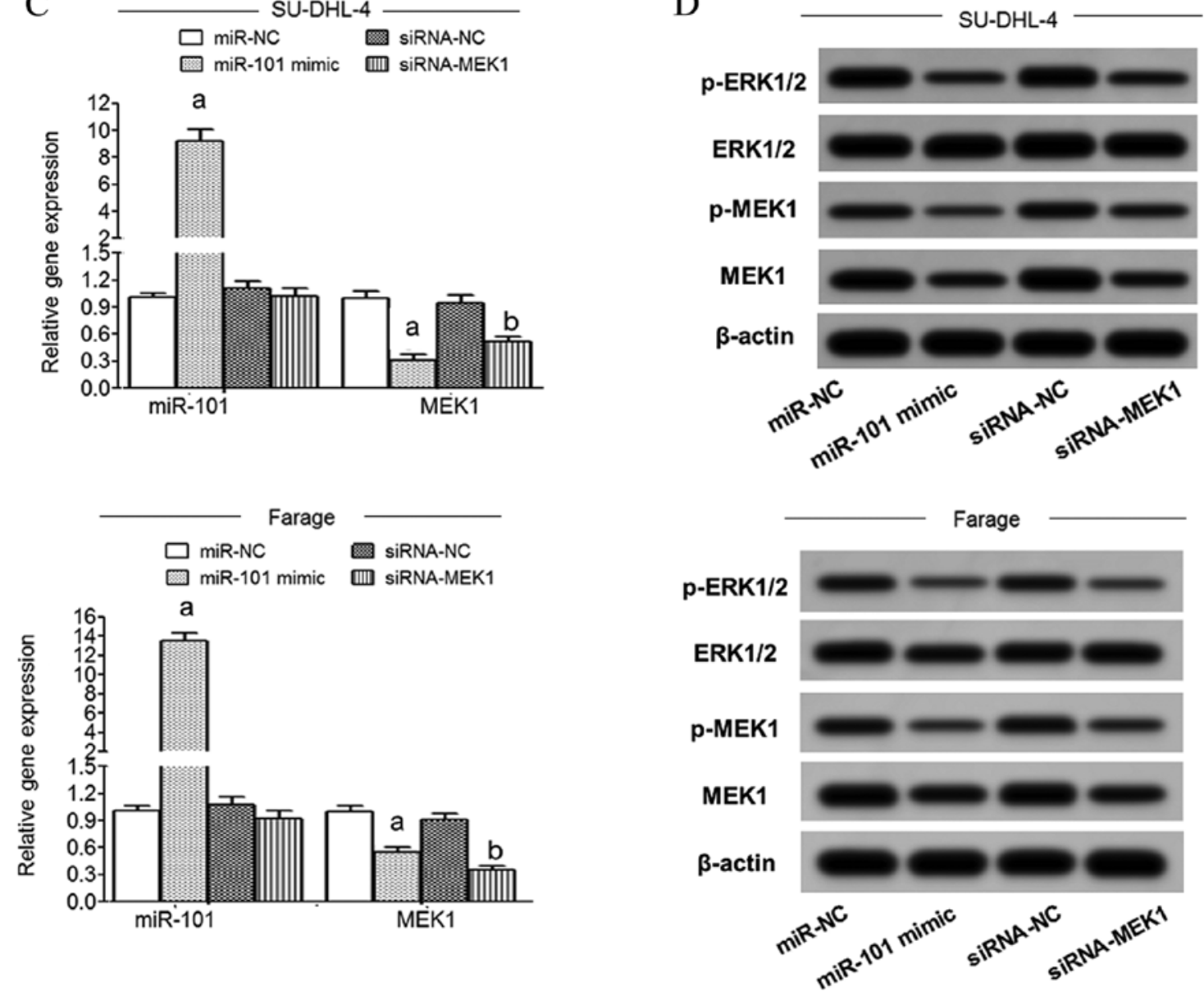

Figure 3. Overexpression of miR-101 downregulates ERK/MAPK pathway activity. (A) qRT-PCR detection of miR-101 and MEK1 gene expression in normal human B lymphoblastoid cell lines (HCC1954 BL and NCI-BL2009) and human DLBCL cell lines (SU-DHL-4 and Farage). ${ }^{\mathrm{a}} \mathrm{P}<0.05$, compared with HCC1954 BL cells; ${ }^{b} \mathrm{P}<0.05$, compared with NCI-BL2009 cells (B) Western blot analysis of p-MEK1 and MEK1 protein expression in the cell lines. (C) qRT-PCR of miR-101 and MEK1 gene expression in SU-DHL-4 and Farage cells transfected with miR-101 mimic and siRNA-MEK1 and the negative controls (NC) (D) Western blot analysis of protein expression of ERK/MAPK pathway-related proteins in SU-DHL-4 and Farage cells transfected with miR-101 and siRNA-MEK1 and the negative controls (NC). ${ }^{a} \mathrm{P}<0.05$, compared to miR-NC; ${ }^{b} \mathrm{P}<0.05$, compared to siRNA-NC. DLBCL, diffuse large $\mathrm{B}$ cell lymphoma.

\section{Discussion}

Lymphomas include Hodgkin lymphoma (HL) and non-Hodgkin lymphoma (NHL), of which NHL is the most common accounting for $>90 \%$ of lymphomas (3). Based on the difference in cell origin, NHL can be divided into B, T, and $\mathrm{NK} / \mathrm{T}$ cell types. The B cell type of NHL is the main type of all NHLs, and the incidence is $>80 \%$ (19). Diffuse large B cell lymphoma (DLBCL) is the most common type of B-NHL, accounting for approximately $50 \%$ of B-NHLs, and accounting for approximately $40 \%$ of all NHL cases $(4,5)$. DLBCL, the most common NHL in adults, is an invasive lymphoma with a high degree of invasiveness, has a poor response to treatment and a corresponding poor prognosis. Therefore, an in-depth study of the pathogenesis of lymphoma is of great significance for screening early diagnostic markers, improving therapeutic effect, and improving the prognosis of patients.

The MAPK signaling pathway is an important signal transduction system that widely exists in eukaryotic cells, and can be mediated and activated by cell factor receptors, 
A
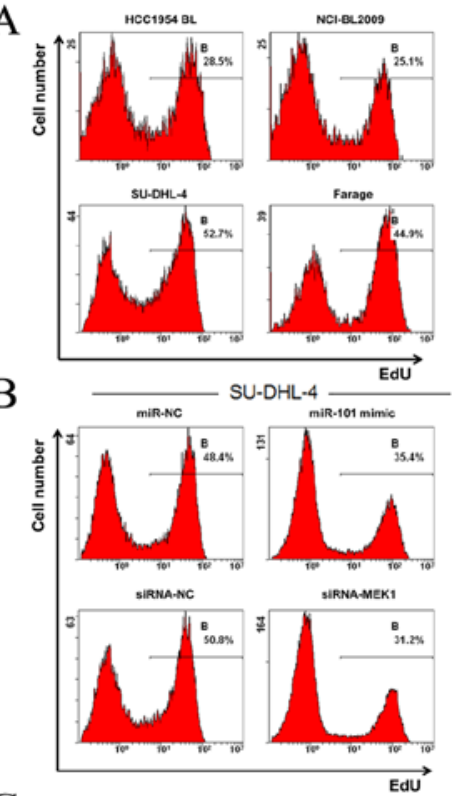

$\mathrm{C}$

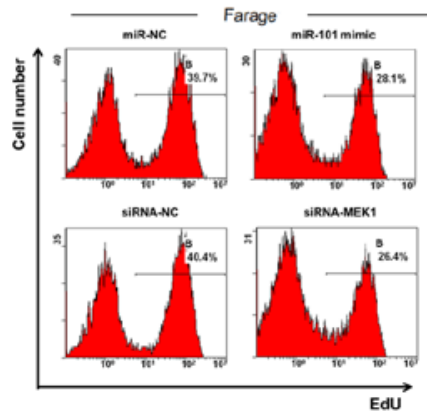

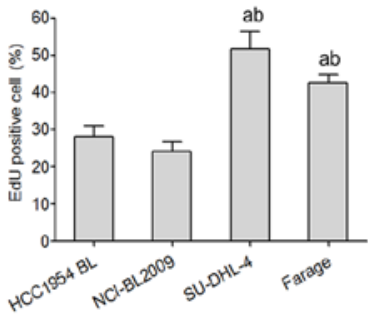
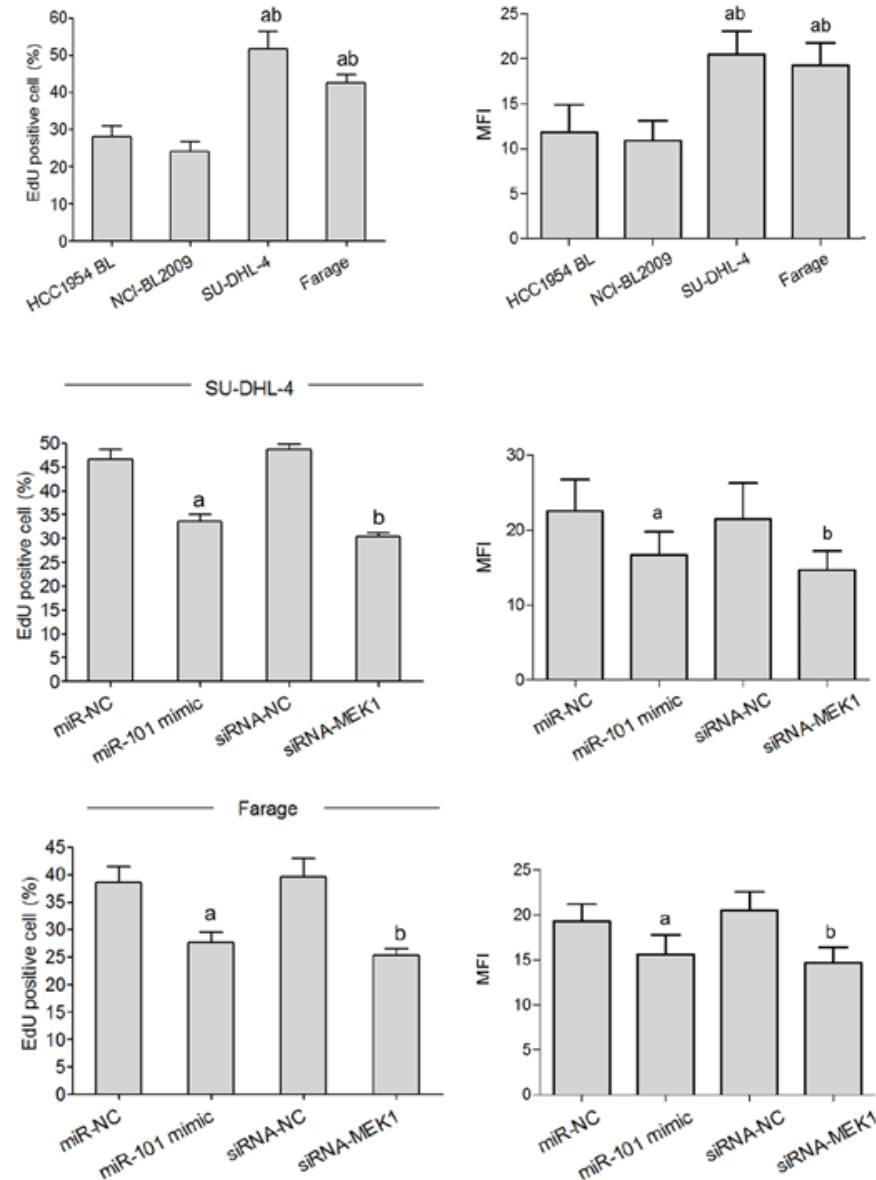

Figure 4. Overexpression of miR-101 inhibits the proliferation of lymphoma cells. (A) EdU staining detection of cell proliferation of normal human B lymphoblastoid cell lines (HCC1954 BL and NCI-BL2009). The percentage of EdU positive cells and its mean fluorescence intensity (MFI) was calculated. ${ }^{a} \mathrm{P}<0.05$, compared with HCC1954 BL cells; ${ }^{\circ} \mathrm{P}<0.05$, compared with NCI-BL2009 cells. EdU staining and flow cytometry was used to detect the proliferation of (B) SU-DHL-4 and (C) Farage cells transfected with miR-101 mimic and siRNA-MEK1 and the negative controls (NC). The percentage of EdU positive cells and its mean fluorescence intensity (MFI) was calculated. ${ }^{a} \mathrm{P}<0.05$, compared to miR-NC; ${ }^{\mathrm{P}} \mathrm{P}<0.05$, compared to siRNA-NC. DLBCL, diffuse large B cell lymphoma.

A

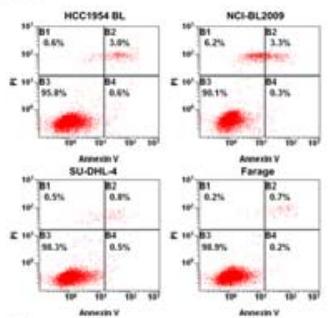

$\mathrm{C}$

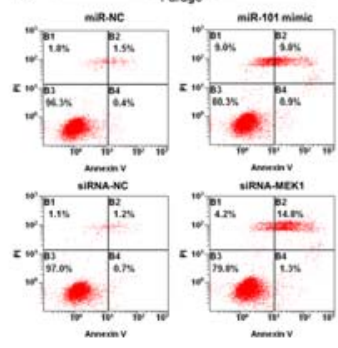

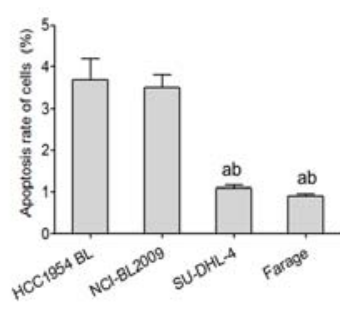

$\mathrm{D}$
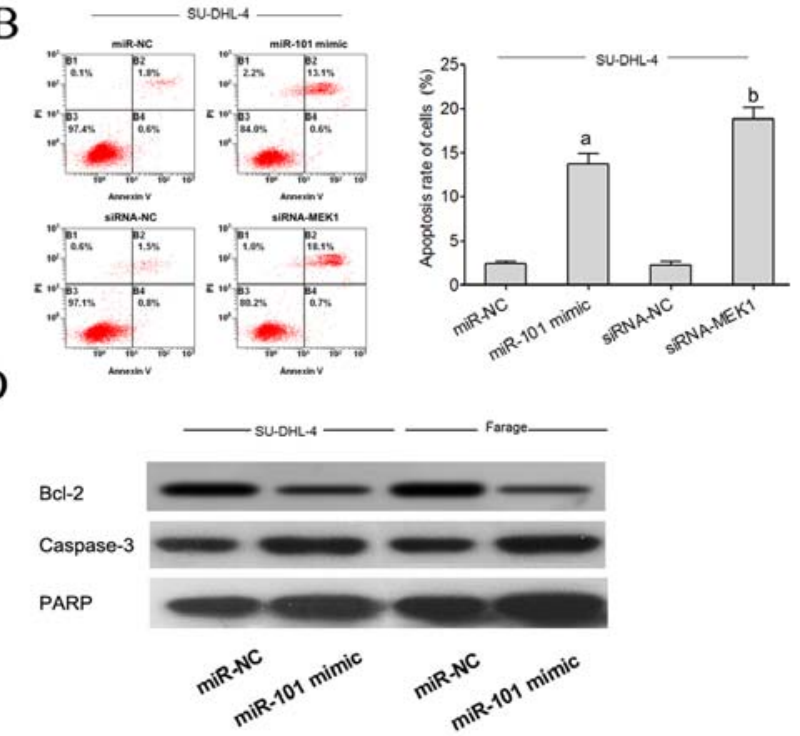

Figure 5. Overexpression of miR-101 promotes the apoptosis of lymphoma cells. (A) Flow cytometric analysis of cell apoptosis of normal human B lymphoblastoid cell lines (HCC1954 BL and NCI-BL2009) and human DLBCL cell lines (SU-DHL-4 and Farage). ${ }^{\text {aP }<0.05, ~ c o m p a r e d ~ w i t h ~ H C C 1954 ~ B L ~ c e l l s ; ~}$ ${ }^{\mathrm{b}} \mathrm{P}<0.05$, compared with NCI-BL2009 cells. Flow cytometric analysis was used to detect the apoptosis of (B) SU-DHL-4 and (C) Farage cells transfected with miR-101 mimic and siRNA-MEK1 and the negative controls (NC). (D) Western blot analysis was used to detect Bcl-2, PARP and caspase-3 expression in SU-DHL-4 and Farage cells transfected with miR-101 mimic and the negative control (NC). ${ }^{a} \mathrm{P}<0.05$, compared to miR-NC; ${ }^{\mathrm{P}}<0.05$, compared to siRNA-NC. DLBCL, diffuse large B cell lymphoma. 
the intracellular receptor tyrosine kinase, $G$ protein coupled receptors, and cytokine receptors in a variety of extracellular stimuli, such as cytokines, growth factors, neurotransmitters, and $\mathrm{G}$ protein coupled receptor ligand interactions. The MAPK signaling pathway consequently activates a variety of transcription factors in the nucleus, regulates various protein kinase reactions in cells, regulates the transcription and expression of target genes, and ultimately affects cell survival, proliferation, migration, apoptosis, and other physiologic and biological processes $(20,21)$. The MAPK signaling pathway family primarily consists of the following 4 pathways: ERK; c-Jun N-terminal kinase (JNK); p38 mitogen-activated protein kinase (p38 MAPK); and large mitogen activated protein kinase pathway (BMK1). Furthermore, the ERK/MAPK signaling pathway mediated by the MAPK signal transduction pathway is the classic MAPK signaling pathway, which plays a major role in the MAPK signaling transduction pathway. The ERK/MAPK signaling pathway is widely expressed in many tissues and cells, and can regulate cell proliferation, the cell cycle, apoptosis, migration and invasion, and many other biological processes $(22,23)$. Excessive activation of the ERK/MAPK signaling pathway can cause abnormal proliferation, apoptosis and differentiation of cells, and thus is associated with tumor occurrence, progression and metastasis, including gallbladder carcinoma (22), esophageal cancer (24), and breast cancer (24-27).

MEK1 is a double-specific protein kinase, which can act upstream of ERK protein and phosphorylate the Tyr/Thr residues of ERK protein, thereby activating the ERK/MAPK signaling pathway (28). Many studies have shown that the abnormal increase in MEK1 expression or functional activity is related to the occurrence, progression, drug resistance and poor prognosis of many types of tumors, such as pancreatic, ovarian and lung cancers (6-8). Moreover, it has been shown that the expression or enhancement of the function of MEK1 may also play an important role in lymphoma (9). The abnormal decrease in expression of miR-101 is associated with the incidence of lymphoma, suggesting a possible tumor-suppressor effect. Bioinformatic analysis showed that there was a target complementary binding site between miR-101 and MEK1. The aim of the present study was to determine whether or not miR-101 plays a role in regulating MEK1 expression, ERK/MAPK pathway activity, and the proliferation and apoptosis in DLBCL cell lines.

The results of the double luciferase gene report showed that the miR-101 mimic can significantly decrease the relative luciferase activity of wild-type pMIR-MEK1-WT transfected into 293 T cells, but the mutant pMIR-MEK1-MUT transfected 293T cell relative luciferase activity was not affected, indicating that there was targeted regulation of the interaction between miR-101 and MEK1 mRNA. The results of the present study showed that compared with the control group (hyperplastic lymph node tissues), expression of miR-101 in DLBCL tumor tissues was significantly decreased, and the more advanced the clinical stage of the patients, the higher the IPI score and the lower the expression of miR-101, suggesting that the abnormal reduction in miR-101 expression was related to DLBCL features. Furthermore, the survival curve also showed that the survival rate of the patients with low miR-101 expression was significantly less than patients with high miR-101 expression.
Compared with the lymph node tissues of the control group, the expression of MEK and p-MEK1 was significantly increased in the tumor tissues of patients with DLBCL. Moreover, the survival and prognosis of patients with low expression of MEK1 mRNA were significantly better than patients with a high level of MEK1 mRNA expression, which was in contrast to the relationship between miR-101 expression and patient survival and indirectly indicated the differential significance of miR-101 and MEK1 for DLBCL.

In a previous study of the relationship between miR-101 and lymphoma, Sasaki et al (29) showed that the decreased expression of miR-101 in the peripheral blood of lymphoma patients may play an important role in upregulating the expression of its target gene, EZH2, and promoting the pathogenesis of lymphoma. Moreover, Ferreira et al (30) reported that compared with reactive proliferative lymph nodes, the expression of miR-101 in tumor tissues of patients with NHL was significantly decreased, suggesting that miR-101 may be a tumor-suppressor gene in lymphoma. Furthermore, Merkel et al (31) showed that compared with normal human CD3T lymphocytes and lymph node tissues of healthy persons, the expression of miR-101 in anaplastic large cell lymphoma in tumor tissues was reduced by $>2$ - to 20 -fold. Leich et al (17) reasoned that the abnormally reduced expression of miR-101 in tumor tissues of $\mathrm{T}(11,15)$ transposition-negative follicular lymphoma may be related to the enhancement of tumor cell proliferation and malignancy. $\mathrm{Ng}$ et al (18) showed that compared with normal lymph node tissues, the expression of miR-101 in tumor tissues and NK tumor cell lines of NK/T cell lymphoma patients was significantly decreased. Although there are many reports concerning the relationship between miR-101 and the pathogenesis of lymphoma, most were $\mathrm{T}$ cell-derived or NK cell-derived lymphomas; however, research concerning the relationship between miR-101 and DLBCL is limited. The results of the present study showed that the expression of miR-101 in DLBCL patients was significantly lower than that noted in the lymphoid tissue, and was correlated with disease stage, IPI score, survival, and prognosis. In addition to lymphoma, the relationship between miR-101 and the incidence of lymphoblastic leukemia has also been reported. Qian et al (32) showed that the expression of miR-101 in the peripheral blood of patients with lymphoblastic leukemia was decreased significantly compared with healthy individuals. In addition, they also found that the expression of miR-101 in the peripheral blood cells of patients with acute $\mathrm{T}$ lymphoblastic leukemia was significantly less than miR-101 expression in the healthy thymus and bone marrow cells. Furthermore, Liu et al (33) showed that the expression of miR-101 in bone marrow tissues of patients with acute lymphoblastic leukemia was abnormally reduced. These studies indicated that the abnormal decrease in miR-101 expression was a contributing factor in the pathogenesis of lymphoma and lymphocytic leukemia; our study findings mutually corroborated and were supported by these studies.

The Bcl-2 gene of B lymphocytoma is an anti-apoptotic factor (34) which plays an important role in the regulation of the mitochondrial-dependent apoptotic pathway. Bcl-2 protein is localized in the endoplasmic reticulum, mitochondrial membrane, and nuclear membrane, and plays a role in anti-apoptosis through a variety of mechanisms. These 
include inhibition of pro-apoptosis mitochondrial cytochrome c, release to the cytoplasm, blockage of the destruction of cell components by oxygen-free radicals, affecting the transmembrane transport of calcium ions, promoting the formation of heterologous two dimers with the pro-apoptotic proteins of the Bcl-2 family, and promoting the localization and distribution of pro-apoptotic factors (35-38). The results of the present study showed that transfection of miR-101 mimic or siRNA-MEK1 significantly reduced the expression of MEK1, p-MEK1 and p-ERK1/2 in SU-DHL-4 and Farage cells. In addition, the expression of the anti-apoptotic factor, Bcl-2, was significantly reduced, lymphoma cell proliferation was significantly decreased, and apoptosis was significantly increased. Qian et al (32) showed that in the process of the pathogenesis of lymphocytic leukemia, miR-101 acts as a tumor suppressor. Moreover, overexpression of miR-101 was found to decrease the proliferation and invasion of Jurkat lymphocytic leukemia cells, significantly increase apoptosis, and increase the sensitivity of the chemotherapy drug, doxorubicin, by targeting inhibition of the Notch1 gene. Ferreira et al (30) found that histone deacetylase inhibitor treatment inhibited the proliferation of Raji cell lymphoma and induced cell cycle arrest, which was accompanied by significantly increased expression of miR-101, suggesting that miR-101 may have a tumor-suppressor role for the reduction of malignant lymphoma cell biological characteristics. Moreover, Merkel et al (31) showed that overexpression of miR-101 inhibited the expression of mTOR, attenuated the proliferation of SR-786 and Karpas-299 cells, induced cell cycle G1 phase arrest, and significantly promoted cell apoptosis. $\mathrm{Ng}$ et al (18) showed that there is a target regulatory relationship between miR-101 and STMN1 in the NK-YS cell line of NK/T cell lymphomas. Furthermore, miR-101 plays a role in downregulating STMN1 gene expression and inhibiting the proliferation of NK-YS cells. The results of this study showed that miR-101 inhibited the proliferation of lymphoma cells and promoted the inhibition of apoptosis of lymphoma cells, similar to the above results. In the study of the relationship between MEK1 and the biological behavior of lymphoma cells, Kumar et al (39) showed that inhibition of MEK1 downregulated the expression of PD-L1 in B cell lymphoma cells, giving credence to the effect of antitumor immunity, which could be a potential target in the treatment of lymphoma. Further detection by Nguyen et al demonstrated that (9) the downregulation of MEK1 by PD184352 or shRNA could significantly enhance the effect of the chemotherapeutic drug, sorafenib, on inducing apoptosis of DLBCL cells, demonstrating that the enhancement function of MEK1 is related to the malignancy of DLBCL cells, while the antagonistic function of MEK1 was found to reduce the malignant characteristics of DLBCL cells. Kumar et al and Nguyen et al $(9,39)$ showed that the enhancement of EMK1 expression or functional activity is a tumor-promoting factor in lymphoma, while inhibiting the expression and function of MEK1 reduces the malignant effect of lymphoma, which is in agreement with our results. At present, the role of miR-155 in regulating MEK1 expression and DLBCL cell proliferation and apoptosis has been rarely reported. The present study confirmed the relationship between miR-101 and MEK1 and their associated function with DLBCL. The present study also confirmed that miR-101 plays an important role in targeting inhibition of MEK1 expression, affecting the activity of the ERK/MAPK pathway, and regulating the proliferation and apoptosis of lymphoma cells.

In conclusion, downregulation of miR-101 is related to the pathogenesis and prognosis of DLBCL. miR-101 overexpression can inhibit the proliferative ability of lymphoma cells and promote cell apoptosis through targeting inhibition of MEK1 expression.

\section{Acknowledgements}

Not applicable.

\section{Funding}

The present study was supported by the Fujian Provincial Natural Science Foundation 2018J01203.

\section{Availability of data and materials}

All data generated or analyzed during this study are included in this published article.

\section{Authors' contributions}

RZ and YH conceived and designed the study. YH, RZ, YZ, LL and XM performed the experiments. YH, YZ and RZ wrote the paper. YH, RZ and LL reviewed and edited the manuscript. All authors read and approved the manuscript and agree to be accountable for all aspects of the research in ensuring that the accuracy or integrity of any part of the work are appropriately investigated and resolved.

\section{Ethics approval and consent to participate}

All of the patients signed informed consent, which was approved by the Ethics Committee of Zhangzhou Affiliated Hospital of Fujian Medical University (protocol no. 2010032).

\section{Patient consent for publication}

Not applicable.

\section{Competing interests}

The authors state that they have no competing interests.

\section{References}

1. Luminari S: Bridging the gap between epidemiology and clinical research in lymphoma. Leuk Lymphoma 54: 1855-1856, 2013.

2. Smedby KE and Hjalgrim $\mathrm{H}$ : Epidemiology and etiology of mantle cell lymphoma and other non-Hodgkin lymphoma subtypes. Semin Cancer Biol 21: 293-298, 2011.

3. Skrabek P, Turner D and Seftel M: Epidemiology of non-Hodgkin lymphoma. Transfus Apher Sci 49: 133-138, 2013.

4. Hamlin PA, Satram-Hoang S, Reyes C, Hoang KQ, Guduru SR and Skettino S: Treatment patterns and comparative effectiveness in elderly diffuse large B-cell lymphoma patients: A surveillance, epidemiology, and end results-medicare analysis. Oncologist 19: 1249-1257, 2014 
5. Castillo JJ, Winer ES and Olszewski AJ: Sites of extranoda involvement are prognostic in patients with diffuse large B-cell lymphoma in the rituximab era: An analysis of the surveillance, epidemiology and end results database. Am J Hematol 89: 310-314, 2014.

6. Gysin S, Paquette J and McMahon M: Analysis of mRNA profiles after MEK1/2 inhibition in human pancreatic cancer cell lines reveals pathways involved in drug sensitivity. Mol Cancer Res 10: 1607-1619, 2012.

7. Pénzváltó Z, Lánczky A, Lénárt J, Meggyesházi N, Krenács T, Szoboszlai N, Denkert C, Pete I and Győrffy B: MEK1 is associated with carboplatin resistance and is a prognostic biomarker in epithelial ovarian cancer. BMC Cancer 14: 837, 2014.

8. Song JY, Kim CS, Lee JH, Jang SJ, Lee SW, Hwang JJ, Lim C, Lee G, Seo J, Cho SY and Choi J: Dual inhibition of MEK1/2 and EGFR synergistically induces caspase-3-dependent apoptosis in EGFR inhibitor-resistant lung cancer cells via BIM upregulation. Invest New Drugs 31: 1458-1465, 2013.

9. Nguyen TK, Jordan N, Friedberg J, Fisher RI, Dent P and Grant S: Inhibition of MEK/ERK1/2 sensitizes lymphoma cells to sorafenib-induced apoptosis. Leuk Res 34: 379-386, 2010.

10. Zheng W, Liu Z, Zhang W and Hu X: miR-31 functions as an oncogene in cervical cancer. Arch Gynecol Obstet 292: 1083-1089, 2005.

11. Wang LQ, Zhang Y, Yan H, Liu KJ and Zhang S: MicroRNA-373 functions as an oncogene and targets YOD1 gene in cervical cancer. Biochem Biophys Res Commun 459: 515-520, 2015.

12. Mou Z, Xu X, Dong M and Xu J: MicroRNA-148b acts as a tumor suppressor in cervical cancer by inducing G1/S-phase cell cycle arrest and apoptosis in a caspase-3-dependent manner. Med Sci Monit 22: 2809-2815, 2016.

13. Fan D, Wang Y, Qi P, Chen Y, Xu P, Yang X, Jin X and Tian X: MicroRNA-183 functions as the tumor suppressor via inhibiting cellular invasion and metastasis by targeting MMP-9 in cervical cancer. Gynecol Oncol 141: 166-174, 2016.

14. Chen LG, Xia YJ and Cui Y: Upregulation of miR-101 enhances the cytotoxic effect of anticancer drugs through inhibition of colon cancer cell proliferation. Oncol Rep 38: 100-108, 2017.

15. Lin C, Huang F, Li QZ and Zhang YJ: miR-101 suppresses tumor proliferation and migration, and induces apoptosis by targeting EZH2 in esophageal cancer cells. Int J Clin Exp Pathol 7: 6543-6550, 2014.

16. Liu L, Guo J, Yu L, Cai J, Gui T, Tang H, Song L, Wang J, Han F, Yang C, et al: miR-101 regulates expression of EZH2 and contributes to progression of and cisplatin resistance in epithelial ovarian cancer. Tumour Biol 35: 12619-12626, 2014.

17. Leich E, Zamo A, Horn H, Haralambieva E, Puppe B, Gascoyne RD, Chan WC, Braziel RM, Rimsza LM, Weisenburger DD, et al: MicroRNA profiles of $t(14 ; 18)$-negative follicular lymphoma support a late germinal center B-cell phenotype. Blood 118: 5550-5558, 2011.

18. Ng SB, Yan J, Huang G, Selvarajan V, Tay JL, Lin B, Bi C, Tan J, Kwong YL, Shimizu N, et al: Dysregulated microRNAs affect pathways and targets of biologic relevance in nasal-type natural killer/T-cell lymphoma. Blood 118: 4919-4929, 2011.

19. Chihara D, Nastoupil LJ, Williams JN, Lee P, Koff JL and Flowers CR: New insights into the epidemiology of non-Hodgkin lymphoma and implications for therapy. Expert Rev Anticancer Ther 15: 531-544, 2015.

20. Pancione M, Giordano G, Parcesepe P, Cerulo L, Coppola L, Curatolo AD, Conciatori F, Milella M and Porras A: Emerging insight into MAPK inhibitors and immunotherapy in colorectal cancer. Curr Med Chem 24: 1383-1402, 2017.

21. Zhang X, Liu K, Zhang T, Wang Z, Qin X, Jing X, Wu H, Ji X, He Y and Zhao R: Cortactin promotes colorectal cancer cell proliferation by activating the EGFR-MAPK pathway. Oncotarget 8: 1541-1554, 2017.

22. Buchegger K, Silva R, López J, Ili C, Araya JC, Leal P, Brebi P, Riquelme I and Roa JC: The ERK/MAPK pathway is overexpressed and activated in gallbladder cancer. Pathol Res Pract 213 $476-482,2017$
23. Liao T, Wen D, Ma B, Hu JQ, Qu N, Shi RL, Liu L, Guan Q, Li DS and Ji QH: Yes-associated protein 1 promotes papillary thyroid cancer cell proliferation by activating the ERK/MAPK signaling pathway. Oncotarget 8: 11719-11728, 2017.

24. Zheng ST, Huo Q, Tuerxun A, Ma WJ, Lv GD, Huang CG, Liu Q, Wang X, Lin RY, Sheyhidin I and Lu XM: The expression and activation of ERK/MAPK pathway in human esophageal cancer cell line EC9706. Mol Biol Rep 38: 865-872, 2011.

25. Chen P, Xu W, Luo Y, Zhang Y, He Y, Yang S and Yuan Z: MicroRNA 543 suppresses breast cancer cell proliferation, blocks cell cycle and induces cell apoptosis via direct targeting of ERK/MAPK. Onco Targets Ther 10: 1423-1431, 2017.

26. Koyama T, Ogawara K, Kasamatsu A, Okamoto A, Kasama H, Minakawa Y, Shimada K, Yokoe H, Shiiba M, Tanzawa H, et al: ANGPTL3 is a novel biomarker as it activates ERK/MAPK pathway in oral cancer. Cancer Med 4: 759-769, 2015.

27. Lu Z, Ding L, Hong H, Hoggard J, Lu Q and Chen YH: Claudin-7 inhibits human lung cancer cell migration and invasion through ERK/MAPK signaling pathway. Exp Cell Res 317: 1935-1946, 2011.

28. Wong KK: Recent developments in anti-cancer agents targeting the Ras/Raf/MEK/ERK pathway. Recent Pat Anticancer Drug Discov 4: 28-35, 2009

29. Sasaki D, Imaizumi Y, Hasegawa H, Osaka A, Tsukasaki K, Choi YL, Mano H, Marquez VE, Hayashi T, Yanagihara K, et al: Overexpression of Enhancer of zeste homolog 2 with trimethylation of lysine 27 on histone $\mathrm{H} 3$ in adult T-cell leukemia/lymphoma as a target for epigenetic therapy. Haematologica 96: 712-719, 2011.

30. Ferreira AC, Robaina MC, Rezende LM, Severino P and Klumb CE: Histone deacetylase inhibitor prevents cell growth in Burkitt's lymphoma by regulating PI3K/Akt pathways and leads to upregulation of miR-143, miR-145, and miR-101. Ann Hematol 93: 983-993, 2014.

31. Merkel O, Hamacher F, Laimer D, Sifft E, Trajanoski Z, Scheideler M, Egger G, Hassler MR, Thallinger C, Schmatz A, et al: Identification of differential and functionally active miRNAs in both anaplastic lymphoma kinase (ALK) ${ }^{+}$ and ALK ${ }^{-}$anaplastic large-cell lymphoma. Proc Natl Acad Sci USA 107: 16228-16233, 2010.

32. Qian L, Zhang W, Lei B, He A, Ye L, Li X and Dong X: MicroRNA-101 regulates T-cell acute lymphoblastic leukemia progression and chemotherapeutic sensitivity by targeting Notch1. Oncol Rep 36: 2511-2516, 2016.

33. Liu X, Zou L, Zhu L, Zhang H, Du C, Li Z, Gao C, Zhao X, Bao S and Zheng $\mathrm{H}$ : miRNA mediated up-regulation of cochaperone p23 acts as an anti-apoptotic factor in childhood acute lymphoblastic leukemia. Leuk Res 36: 1098-1104, 2012.

34. Gahl RF, Dwivedi P and Tjandra N: Bcl-2 proteins bid and bax form a network to permeabilize the mitochondria at the onset of apoptosis. Cell Death Dis 7: e2424, 2016.

35. Jagani H, Kasinathan N, Meka SR and Josyula VR: Antiapoptotic Bcl-2 protein as a potential target for cancer therapy: A mini review. Artif Cells Nanomed Biotechnol 44: 1212-1221, 2016.

36. Um HD: Bcl-2 family proteins as regulators of cancer cell invasion and metastasis: a review focusing on mitochondrial respiration and reactive oxygen species. Oncotarget 7: 5193-5203, 2016.

37. Sivakumar D and Sivaraman T: A review on structures and functions of Bcl-2 family proteins from homo sapiens. Protein Pept Lett 23: 932-941, 2016.

38. Yang D, Chen MB, Wang LQ, Yang L, Liu CY and Lu PH: Bcl-2 expression predicts sensitivity to chemotherapy in breast cancer: A systematic review and meta-analysis. J Exp Clin Cancer Res 32: 105, 2013.

39. Kumar SR, Kim DY, Henry CJ, Bryan JN, Robinson KL and Eaton AM: Programmed death ligand 1 is expressed in canine B cell lymphoma and downregulated by MEK inhibitors. Vet Comp Oncol 15: 1527-1536, 2017. 